\title{
An application of Wilson system in numerical solution of Fredholm integral equations
}

\author{
B. KH. MOUSAVI, A. ASKARI HEMMAT ${ }^{\dagger}$ AND M. H. HEYDARI
}

\author{
Date of Receiving : $\quad 15.05 .2017$ \\ Date of Revision : $\quad 04.07 .2017$ \\ Date of Acceptance : 04.08 .2017
}

\begin{abstract}
A numerical method based on the Wilson wavelets for solving Fredholm integral equations and Fredholm integro-differential (FID) equations is provided. Using Wilson wavelets approximation method Fredholm integral equations is reduced to algebraic equations. Furthermore the operational matrix of integration for Wilson wavelets is obtained. Finally, a numerical comparison between results obtained by Wilson wavelets and CAS wavelets is carried out. The applicability and accuracy of the proposed method are also checked using numerical examples.
\end{abstract}

\section{Introduction}

Integral equations are used as mathematical models in many physical situations. There exist different methods for solving integral equations and recently methods using wavelets have been applied in engineering and sciences[16]. Haar wavelets [3, 13], CAS wavelets [10, 17], Harmonic wavelets [6], Chebyshev wavelets [1, 2, 14], Legendre wavelets $[11,15,18]$ for solving integral and differential equations has produced significant results. In this paper we propose solving integral and differential equations using Wilson wavelets. These wavelets are a special case of orthonormal wavelets with a simple structure and methods based on them are expected to give better results. In order to compute an approximate solution for the second Fredholm integral equations, we first analyze the convergence of Wilson wavelets and then obtain a new operational matrix of integration for the basis functions to eliminate the integral operation and reduce the problem into solving a system of algebraic equations. We also provide illustrative examples to demonstrate the validity and applicability of the method.

The paper is organized as follows: Section 2, contains introducing Wilson wavelets and their properties. In section 3, we present a method to solve the second Fredholm integral equations. In section 4, we present a method for solving FID equations using

2010 Mathematics Subject Classification. 65R20, 45B05.

Key words and phrases. Wilson system, Wilson wavelets, Fredholm integral equation, Integrodifferential equation, Operational matrix of integration.

Communicated by: Suman Panwar

${ }^{\dagger}$ Corresponding author. 